\title{
INDEX NOMINUM
}

[M. Appuleius-] 163

Adams C.E.P. 203

Aemilius Geler 161

Afrique 239, 248, 249, 254, 259, 481-493

Agricola 104

Agrippa 210, 212, 372, 378, 384

Aila (Aqaba) 466-468

Ala Veterana Gallica 244

Albano 255

Alésia 255

Alexandria 97, 451

Alexandria/Nikopolis 537

Antioche 257

Antistius Adventus 361

Antonine Wall 355, 358-360, 363

Antoninus Pius 358-359

Apamea 539, 542, 545

Apolonius Dionysius 173

Appius Celer 152

Aquitania 174, 175

Arabia 439-61

Aragoe 499, 504

Arangio-Ruiz V. 130

Arausio 83

Archelaus 411

Archimedes 267, 273

Armorique 248

Arruntius Paulinus 166

Arruntius Valens, Q. $\quad$ 178, 179

Asclepium 483

Asie Mineure 248

Athenaeus Mechanicus 266

Atilius Sp. f. Vol. Primus, Q. (interprex, centurio, negotiator) 311

Auguste 255

Augustus $\quad 86-7,91,92,93,96,107 f f .$, $171-173,176,317,320,339,371,382$

Aurelian 324, 328, 567-8, 580

Aurelius Rufinus 166

Aureolus 565, 568, 577, 580

Autun 257

Avidius Romanus, T. 264

Babatha 407

Bataver 209, 231

Beloch, Julius 75

Besançon 255
Biton of Pergamum 271, 272

Bostra 439-61

Brand C.E. 130

Breeze D.J. 198, 245

Bretagne 239, 247, 256, 259

Britain 101, 355-70

Brunt, Peter 7, 10, 18, 75-8, 81, 83, 87

Bu Njem 195, 486, 488

Byzantion/Istanbul 529, 536

Caledonii $\quad 364-367,369,370$

Calpurnius Agricola 361

Cantabri 175

Caracalla 364 n. 46, 365, 367-370

Carcani M. 130

Carinus 577, 581

Carnuntum 257

Carus 577, 581-2

Cassius Dio 98, 99, 498, 506

Cassius Severus, L. 152

Cerialis (C. Petillius) 406

Chassenard (Allier) 240

Chatten 210, 229, 380

Cherusci 93

Claude 160, 255

Claudianus, [-]lentius 152

Claudius 92, 93, 101

Claudius II 566-568, 574

Claudius Iulianus, Ti. 161

Claudius Lucilianus 161

Claudius Marcellus, M. (cos. 222, 215, 214, 210, 208) 24 (n. 5), 31

Claudius Nero, C. (pr. 212) 23-25, 29, 31

Claudius Piso, Ti. 160

Claudius Quadrigarius 68

Claudius Terentianus 244

Claudius Tiberianus 244

Clodius Albinus 364-365

Cohortes prétoriennes 245, 255

Commodus 103, 104, 361-364

Constantine 102, 317

Constantius 99

Cornelius Blasio, Cn. (pr. 194) $28 f$.

Cornelius Cethegus, C. (cos. 197) 27-29

Cornelius Gratus, L. 152

Cornelius Lentulus, L. (cos. 199) 26f., 29 
Cornelius Scipio Africanus, P. (cos. 205, 194) 24-26, 29, 31-33, 44

Cornelius Scipio, Cn. (cos. 222) 22-24, 26, 29

Cornelius Scipio, P. (cos. 218) 22-24, 26, 29

Cornouailles 248

Cremona 97

Cutius Aburianus, P. 161

Danube 102, 247, 248

De Neeve 9-10, 19

Dea Roma 100

Didius Julianus 98, 99

Dii campestres 491

Dimmidi 491

Diocletian 283-290, 511, 517, 518

Dioclétien 256

Dionysius 244

Domitian 95, 104

Domitius Ahenobarbus 19

Donnersberg 215, 217, 229

Drusus 202, 210, 213, 232, 380

Dünsberg 209, 210, 230, 231, 380

Dura Europos 195

Dusares 442f., 452 f.

Eck, Werner see subject index s.v. Bar Kokhba revolt

Éduens 253

Égypte/Egypt 244-245, 281-291

Elusa 467,469

Emporion (Greek town) 37f., 41f., 44, 44 (n. 72)

Erdkamp P. 202

Espagne 248, 254, 259, 260

Favorinus 91

Flaviens 256

Flavius Silva (L.) 395, 402

Flotte d'Alexandrie 244

Flotte de Misène 255

Flotte de Ravenne 255

Forum Iulii 172-174

Fregellae $\quad 70-73$

Gabba 17-18, 142, 143

Gadir/Gades 41, 45

Gaius (Caligula) 101, 104

Galerius 100

Gallienus 512, 520, 569-70, 572-5, 582

Gaule 250, 252, 255, 256

Gavius Fulvius Tranquillus, Q. 157, 158

Gavius Proculus, Q. 154

Gaza $465-467$
Gemellae 490

Génie de Gholaiae 487

Génie de la vexillatio 487

Génie militaire 483

Gentilius Victor, C. (negotiator gladiarius) 308,312

Germanicus 93

Germanie(n) 207-38, 245, 248, 250, $253,254,257,371-90$

Ghassanids 419, 423

Hadrian 91, 101, 102, 103, 355-6, 358, 359

Hadrianoutherae 102

Haltern 374-376, 380, 382, 387

Hauran 443, 448f.

Hedius Verus, C. 154

Heidetraenk-Oppidum 230

Helvius, M. (pr. 197) 33

Herculaneum 343, 351

Hercules 565, 571-3, 575

Herod (the Great) 410,413

Herod Agrippa II $\quad 414,415,417$

Herodian 498, 505

Honorius 419

Hopkins K. 77, 198

Humayma 452f.

Inchtuthil 250, note 62

Isis et Sérapis 484

Italians $\quad 79-80$

Iulius Antiochus, Gaius 179

Iulius Arrianus, C. 162

Iulius Arrianus, T. 162

Iulius Demetrius, Gaius 179

Iulius Faustinus, P. 276

Iulius Glutacus, Tiberius 174

Iulius $\mathrm{Ho}$ [noratus ?] 151

Iulius Proculus 166

Iulius Titianus, Gaius 179

Iulius Victor (negotiator vestiarius) 304

Iunius Silanus, M. (pr. 212) 25f., 29, 31

Jawf 442,451

Jerusalem 451f.

Jews 393-420

Josephus, Flavius 97, 394, 402-405, $410,412,414,416$

Judaea 393-407

Julian (= Julianus) 99, 100

Julius Alexander, Tiberius $\quad 410,416,417$

Julius Caesar 417

Julius Marcus, C. 370

Jupiter 487,492 
Jupiter Dolichenus 489, 490

Jupiter Hammon 488

Kastron Mefaa 475

Köln 213, 217, 224, 228, 233-36, 372-374, 376-379, 384-390

Kyrenaika 450

Lambèse (= Lambaesis) 481, 482, 483, 485

Lauriacum 257

Legio I Mineruia 255

Legio II Parthica 255, 537-38

Legio II Traiana fortis 247, 537

Legio III Augusta 102, 254 (n. 87)

Legio III Cyrenaica $446 \mathrm{ff}$.

Legio III Gallica 421

Legio IV Scythica 421

Legio XXX Vlpia 255

Lejjun 476

Lepidius Proculus, L. (centurio, benefactor salinatorum Morinorum et Menapiorum) 306

LeRoux P. 199-200

Liberius Victor, M. (negotiator frumentarius) 306

Livy (= Livius) $47-74$

Lo Cascio E. 75, 87

Lucretius Marcellus 166

Lucullus 95

Luttwak, Edward 401-403, 405

Mainz 209, 213, 227-236, 387

Malagbel 492

Mamertini 570

Manlius Acidinus, L. (pr. 210) 26-29

Marc Antony (Marcus Antonius) 171, 173

Marcius, L. 45

Marcus Aurelius 356, 360-362, 500, 511,516

Marius 65-66

Marktbreit 233, 234

Mars Canaphar 488

Martberg 215, 217, 221

Marx K. 142

Masada 395, 400-404

Matutinius Maximus, M. (negotiator sagarius) 308-309

Maximinus Thrax 505

Messius V[-], L. 155

Mithraea 485

Monceaux-le-Comte (inscription) 253

Neapolis 103

Neptune (= Neptunus) 484
Nero (= Néron) $103,104,256$

Nessana 469-472, 477

New Carthage (= Carthago Nova) 38

Nijmegen 93, 373, 376, 383

Nitl 475-477

Nonienus Pudens, Q. 253

Nonius Datus 262

Oboda (Avdat) 465, 468, 469, 471-473, 478,480

Octavius (Tab. Vind. 2.309) 306

Ofilius Theodorus 156

Onasandros 181-191

Oppius Marcellinus, P. 155

Pacideius Carpianus, L. 155

Paeligna/i 48-49, 57, 65-6

Palmyra 421, 454

Panopolis 283-290

Pannonie 243

Paris 99

Parthians 96

Passerini A. 142

Paul (= Paulus) 410, 411

Pelusium 450

Pertinax 98

Petra 465-467

Philip the Arabian (= Philippus Arabs) 504-505

Picenum 19

Picts 365-366

Pisacane C. 130

Pline l'Ancien (= Plinius Maior) 249

Pliny the Younger (= Plinius Minor) 196

Plutarch (= Plutarchus) 61-64

Pollard N. 202, 206

Polybius 47-74

Pompeii 343, 344, 345, 348, 349, 350, 351

Pompey (= Cn. Pompeius Magnus) 19

Porcius Cato, M. (cos. 195) 42, 44, 46

Poseidonius 63

Pydna 49, 56, 61-64

Quinctilius Varus 210, 212, 375, 383, 387, 388

Raphanaea 421

Rathbone D. 17

Remesal Rodríguez J. 197

Rhénanie 253

Rhine 94

Rich J. 4

Rome, castra Misenatium $\quad 320,330$ 
Rome, castra nova equitum singularium 318, 324, 331

Rome, castra peregrina 323

Rome, castra praetoria $\quad 323-330$

Rome, castra urbana 320

Rome, city of $\quad 95-98,100-105,255$, 529-30, 536, 538-39, 542-43, 545

Rome, military camps in 315-341

Rome, military sepulchral monuments $\quad 332-338$, cf. 529-45

Rome, military units in 315-341

Rome, stationes cohortium vigilum 320

Rosenstein N. 6, 11-12, 15

Rosselli N. 130

Roth J. 202

Rusonius Secundus, C. (negotiator sagarius, sevir augustalis) 308

Rutilius Rufus, P. 80

Sallust (= Sallustius) $\quad 65-6,73$

Salonica 100

Samaritans $411,413,417$

Sappius Flavus, C. 167

Scipio Nasica 63

Sempronius Gracchus, Tib. 3-4, 12

Sempronius Tuditanus, C. (pr. 197) 33

Septime Sévère $(=$ Septimius Severus) 246, 255

Septimius Severus 96, 98, 99, 100, 324, 336, 341, 364-370, 502-5, 513, 527

Servilius Diodorus, C. 163

Severan Emperors 504, 506

Severina 574

Sisenna 265

Skaptopare 499

Sobota (Shivta) 465, 469, 471-473, 478

Sol Yarhibôl 490

Spain 21-46, 497

Stern M. 405-406

Stertinius, L. $28 \mathrm{f}$.

Suetonius 103

Sulla, son of Sennus (nummularius) 310

Syria 421-436

Syria, basalt hills and desert fringe 421-436

Syria, cities in $421-436$

Syria, Diocletianic system 422

Syria, epigraphic habit in $\quad 425-437$

Tacitus $92,97,104,262,278,279$

Takina 499

Tarraco 38,44

Taubenschlag R. 130

Tchernia A. 203
Terentianus 177

Tetrarchy 100

Thmouis 500

Tiberius 92, 174, 211, 212, 213, 320, 339,371

Tiberius Gracchus (= Tib. Sempronius Gracchus) 3-4, 12

Titelberg 216, 217, 218, 221

Titus 97, 98, 416

Tongeren $372,374,376,378-379,383$, 385, 389

Trajan (= M. Ulpius Traianus) 93, 102, 355

Treverer, Civitas Treverorum 209, 214-221, 224-227, 230, 231

Trèves 257

Trier 209, 219-227, 236, 237

Ubiens 253

Ubier (= Ubii) 209, 210, 384-9

Ulpian (= Ulpianus) 176

Ulpius Marcellus 361-363

Umm al-Rasas (Mefa) 474-477

Valerius Antias 56, 60, 62, 64, 67-74

Valerius Oc(u)latius 152

Vegetius 177, 266, 267, 277

Velleius Paterculus 69

Vendrand-Voyer J. $\quad 132,142$

Veranius, Q. 182

Versenus Aper, L. 153

Versenus Granianus 153

Vespasian/Vespasien $(=\mathrm{T}$. Flavius

Vespasianus) 97, 99, 100, 254, 417

Vettius Latro, M. 163

Vibius Quartus, G. 160

Victoria 98

Victorius Regulus (negotiator purpurarius) 304-305

Viduc(i)us, A. $\quad 307-308,312$

Vindolanda 195, 201, 202, 203, 204, 306

Vindonissa 195

Vitruvius 262, 265, 266, 270, 272, 273

Waldgirmes 210, 229, 375-376, 378, 380-383, 389

Whittaker C.R. 204

Wierschowski L. 201

Yajuz 459

Yotvata 466, 467

Zenobia 574

Zeus-Ammon/Iuppiter Hammon 450ff. 